\title{
Religiosidad y cerebro: Las funciones neuropsicológicas en personas creyentes ${ }^{i}$ \\ Religiosity and Brain: Neuropsychological Functions in Believers
}

\section{Por:Diego Alejandro Calle Sandoval'\& Héctor David Arcila Ayala ${ }^{2}$}

1. Doctor en Psicología con orientación en neurociencias cognitivas aplicadas; magister en filosofía de la Universidad del Valle. Psicólogo de la Universidad del Valle. Orcid: http://orcid.org/0000-0002-4917-5819 Scholar: https://scholar.google.es/citations?user=S5e-En8AAAAJ\&hl=es\&oi=ao Contacto: Diegoa.calle@unilibre.edu.co

2. Maestrando en Filosofía de la Universidad Pontificia Bolivariana; especialista en Cerencia Educativa de la Corporación Universitaria Minuto de Dios; Licenciado en Educación Religiosa de la Universidad Pontificia Bolivariana, docente adscrito al Centro Interdisciplinario de Estudios Humanísticos CIDEH de la Universidad de San Buenaventura. Orcid: https://orcid.org/0000-0002-3800-2277 Contacto: hector.arcila@usbmed.edu.co

\section{G OPEN ACCESS}

\section{(C) $\underset{\mathrm{BY}}{\mathrm{BV}} \mathrm{ND}_{\mathrm{NC}}$}

Copyright: (C) 2020 El Ágora USB.

La Revista El Ágora USB proporciona acceso abierto a todos sus contenidos bajo los términos de la licencia creative commons Atribución-NoComercial-SinDerivar 4.0 Internacional (CC BY-NC-ND 4.0)

Tipo de artículo: Reflexión Recibido: septiembre de 2019

Revisado: octubre de 2019

Aceptado: enero de 2020

Doi: 10.21500/16578031.5145

Citar así: Calle Sandoval, D., \& Arcila Ayala, H. (2020). Religiosidad y cerebro: Las funciones neuropsicológicas en personas creyentes. El Ágora USB, 20 (2). 298-304

Doi: $10.21500 / 16578031.5145$

\section{Resumen}

La neuropsicología de la religión permite una apertura científica a la explicación del fenómeno religioso y su impacto en el psiquismo, el presente documento intenta una reflexión que da cuenta de esa estrecha relación e influencia que ejerce la práctica religiosa en la salud mental y en el desarrollo de funciones neuropsicológicas en las personas. Dicha relación, se fortalece con los descubrimientos contemporáneos de la neurociencia cognitiva y la antropobiología.

Palabras clave: Neuropsicología; funciones ejecutivas; empatía; Psicología de la religión.

\section{Abstract}

Neuropsychology of religion allows a scientific openness to the explanation of the religious phenomenon, and its impact on psychism. This document attempts a reflection, which gives an account of this close relationship, and influence exerted by religious practice on mental health and the development of neuropsychological functions in people. This relationship is strengthened by contemporary discoveries of cognitive neuroscience and anthropology.

Keywords: Neuropsychology; Executive Functions; Empathy; and Psychology of Religion. 


\section{Introducción}

La paleoantropología contemporánea considera que nuestra especie tiene aproximadamente entre 100 y 200 mil años de existencia (Arsuaga \& Martínez, 1998). De estos, solo los últimos 20040 mil arrojan evidencia de prácticas culturales religiosas, mitológicas y cosmogónicas (Coppens, Grolier, Pelicier, Reeves \& Reisse, 1988; Willson, 2012; Calle, 2012). Así pues, muchos antropólogos afirman que las religiones emergen a partir de la necesidad individual, ya que desde tiempos antiguos nuestros antepasados, a través de prácticas y rituales, demostraban ese vínculo y conciliación creado con un ser superior (Lima, 2008). Incluso hoy, se estima que en el mundo más de un $85 \%$ de las personas practica alguna religión y de estas 2 millones se reconocen cristianas según datos de la última década (Zegarra-Valdivia \& Chino, 2016).

Varios académicos consideran el fenómeno religioso como una manifestación del espíritu, que junto a la inteligencia permite a cada persona tomar las creencias y prácticas de cada credo como propias en su vida (Lima, 2008). En este sentido, la religión tiene relación directa con la toma de decisiones del ser humano, pues involucra creencias, el sentimiento y conjunto de normas que rigen el comportamiento humano.

\section{Espiritualidad y fenómeno religioso}

La espiritualidad, pues, es la que más allá de las creencias mueve al ser humano a ejercer toda práctica religiosa. En palabras de Estrada (1992), se puede definir espiritualidad como la vida según el espíritu, es decir, la forma de vida que se deja guiar por el espíritu de Cristo; podríamos decir, entonces, que la espiritualidad es la fuerza motivacional que impulsa al ser humano a dejarse encaminar por la religión o el ser superior en quien depositan su fe.

Se podría decir que la religión juega un papel muy importante en la sociedad, esta genera una gran influencia en los seres humanos y en su comportamiento, de cierta forma genera un control social, puesto que los seres humanos actúan de acuerdo a sus creencias. De acuerdo a Durkheim (1915, p.38), "las religiones representan sistemas coordinados de creencias y prácticas específicas que definen lo sagrado - esto es, prescriben un orden sobre ciertos fenómenos o elementos cuya existencia tiene lugar en un más allá, fuera de la vida ordinaria". Es decir, la religión origina un régimen de creencias que dan explicación a la sociedad sobre ciertos acontecimientos que no tienen una explicación científica. La religión marca, de cierta forma, un sistema de reglas y creencias que ayudan a mantener un orden social, pues enmarcan ciertos límites o fronteras entre lo sagrado y lo prohibido: para ser más claros, nos dan la pauta de lo que es permitido hacery lo que no. Es por ello que definen un sistema de creencias dentro de la organización en la cual se congregan; para una mejor comprensión, se hace necesario dar una aproximación al significado de lo que la religión determina iglesia: "Una Iglesia consiste de un conjunto de creencias específicas las cuales son enunciadas por sus líderes y aceptadas por sus adherentes que en su totalidad se reconocen como una religión" (Mundigo, 2005); la iglesia cuenta con un dirigente -o más propiamente dicho-con un líder religioso, quien es el que marca la pauta y da a conocer la reglas que se deben cumplir dentro de este grupo religioso; del mismo modo, las personas que hacen parte del grupo religioso aceptan y adoptan las reglas que la religión determina. Desde este punto de vista, la religión, de cierta forma, ejerce un gran poder o una gran influencia en la vida de las personas que la ejercen, pues como ya se había mencionado, esta determina ciertas pautas de lo que se debe hacer y lo que no, y del mismo modo establece cierto orden dentro de sus congregantes.

Por otra parte, es apropiado dar una explicación del por qué la religión atrae tantas personas y que es lo que les aporta para que estas permanezcan fielmente en pro de la misma. Por esta razón se darán descripciones de las posibles razones por las cuales existe una gran influencia de la religión sobre la sociedad. 
En primer lugar, existe una fuerte relación o influencia de la religión en cuanto a la salud de las personas, dado que las personas acuden a su religión frente a cuestiones relacionadas con su salud o enfermedad y que esta tendencia no desaparece ni siquiera con el descubrimiento y la evolución de tratamientos médicos cada vez más eficaces y precisos (Moreira-Almeida, Koeing \& Luchetti, 2014). Existe la creencia en las sociedades, desde mucho antes que la ciencia surgiera, que existe una fuerza superior que tiene el poder de curar cualquier mal que aqueje al ser humano y devolver su salud, y que todo esto se puede lograr según esta creencia a través de la religión. No obstante, aunque la ciencia haya adquirido gran poder, ha sido difícil derrotar esta creencia, pues existe mayor influencia de la religión.

Desde que Byrd (1988) diera a conocer sus hallazgos positivos en un grupo de pacientes de una unidad de cuidados coronarios, quienes fueron motivo de oración por un grupo de creyentes, sin conocimiento de ellos ni del personal médico y paramédico que los atendía, y se les comparara con otros que recibieron los cuidados habituales en tales casos, reportándose en los primeros mejores resultados sobre su evolución que en los segundos, la oración intercesora, como se le denomina a tal acción, ha pasado a constituir dentro del contenido Religión-salud uno de los más "intrigantes y merecedores de cuidadosa consideración” (Thorensen \& Harris, 2002) y esta es una de las principales razones por la que la religión ha venido tomando fuerza en los últimos años, pues existe la creencia de que esta práctica va más allá de lo científico y de cierta forma, si lo relacionamos con otros casos, en pacientes que practican la espiritualidad genera una modificación en la psiquis que hace incluso que el tratamiento surja mejores resultados.

Según Carrasco (2015), las creencias religiosas tienen el poder de regular conductas tan vitales como la alimentación, y ejerce un gran poder sobre la capacidad que tiene el ser humano para controlar conductas tales como el consumo de sustancias psicoactivas, entre otras. Es tan fuerte el poder que ejercen las distintas religiones que incluso regulan conductas tan vitales como la alimentación, y uno de los casos en los que esta se ve regulada es el hecho de que algunas religiones hasta prohíban comer algunos alimentos como la carne: es el caso de los griegos, los budistas y los hindúes; el hecho de que la religión tenga limitaciones incluso con la alimentación hace también que se limiten las relaciones sociales, pues por sus creencias evitarán de cierta forma relacionarse con personas que no adopten su comportamiento. Esta influencia tan evidente que ha generado la religión en las personas también ha traído consigo fuertes consecuencias negativas, pues aunque poco se hable de esto ha habido personas perjudicadas de cierto modo por estas creencias mal manejadas y es el caso de los denominados "ateos", es decir, las personas que han manifestado su poca o ninguna credibilidad en la existencia del ser supremo al que la religión denomina Dios:

La discriminación social de los ateos ha llegado hasta tal punto que se ha comparado con la que sufren otros grupos minoritarios marginalizados. Algunos ejemplos son: ser asaltado físicamente, sufrir amenazas, el rechazo por parte de familiares, la negación del empleo o la pertenencia a organizaciones comunitarias y otras más, tan graves como la negación de tratamiento hospitalario, o la negación de la custodia de los hijos tan sólo por declarar su ateísmo (Carrasco, 2015).

\section{Religiosidad y Salud Mental}

Es importante hablar de la relación que tiene la religión y la salud mental, pues antes se hablaba poco del tema, al punto de considerar el tema de la religión como algo insignificante para abordar a nivel investigativo. Sin embargo, es importante destacar que autores citados por Sinkim (2017) de gran importancia para los avances que ha presentado la salud mental y que han tenido presente este concepto tan importante para la humanidad; Freud (1979), por ejemplo, considera que la religiosidad contribuye a la domesticación de los instintos destructivos. Es decir que la religión contribuye en la reducción de dichos instintos destructivos en el ser humano, llevando a que estos se vuelvan más adaptativos para la vida humana cotidiana. 
Con el paso del tiempo, la religión es un tema que ha ido tomando gran importancia progresivamente tanto para psicólogos como psiquiatras e investigadores del tema. Esto muestra que la religión está ejerciendo una gran influencia en la vida de las personas, específicamente en su salud mental, puesto que investigadores del tema han centrado su interés en la investigación de la relación o influencia que tiene esta en la salud mental. Zegarra-Valdivial \& Chino (2016) afirman que la religión influye en nuestras convicciones y emociones, operando como un mecanismo de control e interpretación cognitivo de las activaciones neurovegetativas ante la toma de decisiones. Es decir, la religión opera como un instrumento de mediación entre las emociones, nuestro credo e ideología, de cierto modo ejerce un control sobre las decisiones que a diario nos vemos expuestos a tomar es por ello que podremos decir que esta ejerce una gran influencia sobre la toma de decisiones del ser humano y por ende en los procesos mentales del mismo.

Del mismo modo, podríamos decir también que la religión no solo ejerce influencia sobre la toma de decisiones, sino también en la capacidad de reponerse ante eventos traumáticos por los que el ser humano se vea enfrentado; en este caso, estaríamos hablando de que la religión tiene que ver con la resiliencia que el ser humano tenga ante los eventos a los cuales se vea enfrentado:

En un estudio realizado en 199 mujeres maltratadas en la ciudad de Medellín, en Colombia, concluyen que la resiliencia y la espiritualidad son factores que contribuyen al autocuidado y la adaptación exitosa pudiendo ser valiosas en las intervenciones en violencia familiar (Humphreys, 2004; Senter \& Caldwell, 2002).

Así, podríamos decir que la espiritualidad resulta siendo una ayuda importante a la hora de iniciar un proceso terapéutico ante alguna situación traumática, pues es evidente, según el estudio mencionado anteriormente, que brinda estrategias para el afrontamiento de la situación de una manera adecuada, y fomenta una adaptación al proceso brindando herramientas, tales como el auto cuidado e incluso el fortalecimiento del autoconcepto. Es decir, que la religión tiene un gran dominio sobre las funciones neuropsicológicas del ser humano, regulando en gran medida dichos procesos. Es importante resaltar que la espiritualidad ejerce un gran impacto sobre la psiquis del ser humano, puesto que como ya lo habíamos mencionado regula muchos procesos neuropsicológicos, brindando un gran aporte al mismo. Según un estudio realizado en adultos mayores con depresión, se encontró un efecto sobre los síntomas depresivos de estas personas, en donde se evidenció una menor presencia de síntomas depresivos sobre aquellas personas que llevaban una adecuada espiritualidad, destacando una creencia religiosa positiva, es decir, una conexión con un dios benevolente; en el caso de los adultos mayores con una creencia religiosa negativa, es decir, la concepción de un dios castigador, se encontró un aumento de síntomas depresivos, en donde esta espiritualidad, más que ser una ayuda para sus síntomas, reforzaba los mismos (Flores, Huerta, Herrera \& Alonso, 2012).

Desde este punto de vista, la espiritualidad puede ser de gran ayuda para el ser humano y su salud mental en el manejo de patologías tales como la depresión, pero también puede ser un factor negativo para el tratamiento de estas patologías, pues su concepción negativa de la espiritualidad conlleva a un incremento de los síntomas. Es importante aquí destacar que la espiritualidad puede ser un factor contribuyente a la salud mental, pero según lo analizado, puede contribuir de manera positiva o negativa: todo depende de la conexión y de la concepción que tenga el paciente acerca de su dios, es decir, un dios benevolente o castigador.

En un estudio realizado a personas con trastorno de ansiedad se encontró que "De 8 estudios experimentales, 7 (88\%) reportaron una disminución de la ansiedad luego de intervenciones religiosas o espirituales mientras que de los 25 estudios clínicos 17 (64\%) reportó una disminución de la ansiedad luego de intervenciones religiosas o espirituales". Es decir, que desde la práctica religiosa existe una fuerte correlación en la disminución de trastornos psicológicos tales como la ansiedad y 
la depresión; es por ello que es significativo destacar la importancia religión/espiritualidad para la ciencia, específicamente para la psicología y psiquiatría en el tratamiento de patologías psicológicas y psiquiátricas.

En cuanto a la capacidad que tienen las personas para sobreponerse ante una situación difícil, como ya se había mencionado, la religión tiene gran influencia en este aspecto, pues es importante ser reiterativos en que brinda muchas estrategias para que el ser humano pueda sobreponerse ante crisis vitales y accidentales, en donde se ha propuesto incluso y se ha investigado que la lectura de las escrituras juega un papel significativo, pues le enseña a las personas que adoptan esta doctrina de vida, que las adversidades suceden porque su ser supremo así lo dispuso. Es aquí donde se encuentra una modificación en la psiquis, permitiendo que las personas asuman de una manera más adaptativa la adversidad, permitiendo incluso un mejor afrontamiento en la misma e incluso llegando a la mejora (Okulicz-Kozaryn, 2010 citado por Simkin, 2017).

\section{Neurociencia de la empatía y conducta social}

Las neurociencias sociales en la actualidad definen la teoría de la mente (TOM) como una emergencia biológica en la que se suceden procesos que tienen por objeto el reconocimiento metacognitivo de sí mismo, de los otros sujetos y principalmente de sus intenciones, para así, lograr anticipar y realizar un ajuste al propio comportamiento; según lo estiman algunos autores, la TOM, el procesamiento emocional, el sesgo atribucional y la percepción social los pilares de la cognición social (Calle, Grañana, Jiménez \& Romero, 2018).

La TOM como constructo nace en las investigaciones y publicaciones de Premack y Woodruff, (1978) los cuales, a partir de investigaciones en etología describieron el comportamiento empático en primates, conclusiones que serían confirmadas en trabajos biológicos y etológicos por medio de la teoría de las neuronas espejo (Rizzolatti \& Craighero, 2004) y análisis desde los componentes de la cognición social (Calle, 2017).

La empatía o teoría de la mente en el contexto de las neurociencias sociales es una variable de análisis en la que se observan condiciones tanto ontogénicas del sujeto pero también filogenéticas, ya que el medio incide en la composición estructural del comportamiento altruista, sobre ello, la religiosidad como capacidad mentalizadora propia del ser humano, es la expresión de dicha influencia, así lo han hipotetizado Ments, Roelofsma y Treur (2018) quienes mediante un modelo de red temporal-causal, han estimado la importancia del apego y la religiosidad transmitida por los padres y su influencia en la capacidad empática y altruista del sujeto religioso. Desde esta perspectiva, es plausible considerar que la conducta y pensamiento de tipo religioso y moral en la especie humana está arraigada en los aspectos filogenéticos y etológicos de la reciprocidad como base del comportamiento pro-social propio de los primates (Tomasello, 2016).

\section{Neuropsicología de la religiosidad}

En una investigación realizada en colegios de Arequipa, Perú, se hizo la comparación entre estudiantes de edad similar con un alto énfasis de educación religiosa y otras instituciones con menor énfasis en este tipo de educación y se pudo observar que Los estudiantes de la institución parroquial tomarían decisiones más adaptativas, menos impulsivas y con mayores recompensas a largo plazo, mientras que los adolescentes de las otras instituciones, mostrarían una tendencia negativa en sus elecciones, siendo más impulsivos, y con mayores «ansias» de reforzadores a corto plazo. Esto los Ilevaría a tener decisiones de riesgo (Zegarra-Valdivia y. Chino Vilca, 2016); podríamos decir que, según la investigación, la espiritualidad o la religión en términos precisos contribuyen a procesos de adaptación, manejo de impulsividad, mejora en la toma de decisiones, lo que, en término de recompensas para el ser humano a largo plazo, lo llevaría a tener mejores beneficios que aquellos que no realizan esta práctica. Es preciso mencionar que, en este caso, se pudo evidenciar 
(como ya se había descrito) que la religión actúa como mediadora en la toma de decisiones, logrando reducir de manera significativa decisiones riesgosas para el ser humano, pues al regular la impulsividad también mejora en gran medida la toma de decisiones en el mismo, logrando que estas sean beneficiosas y adecuadas para las personas. Dicha capacidad de tomar decisiones es clave en la salud mental de los individuos (Calle \& Gonzáles, 2016).

\section{Conclusiones}

La religión está mediando de manera tan significativa en las personas que practican la espiritualidad, que podríamos decir que limitan todas sus conductas, de modo que estas vayan acorde a lo que su religión proponga; es decir, los seres humanos que practican la espiritualidad limitan su cognición y guían sus conductas de acuerdo a las pautas que están enmarcadas por el reglamento de su religión, esto de cierto modo los acoge y los mantiene en cierto límite que les impide incluso tomar acciones o decisiones riesgosas para ellos, pues en cierta medida, como lo mencionábamos inicialmente, la religión, por medio de sus líderes religiosos, dispone un orden dentro de la misma. Esta racionalidad o cognición limitada, inhibe procesos neuropsicológicos tales como la impulsividad que podrían llevar a las personas a cruzar la frontera que ellos enmarcan y llevarlos al riesgo.

Por otra parte, Martínez (2008) pudo evidenciar que la relación directa con la religión o la práctica de la espiritualidad ayuda a la rehabilitación de pacientes con traumatismo cerebral, en la cual se pudo constatar que los pacientes que practicaban la espiritualidad presentaban una mejor rehabilitación. Cencini y Maneti (2015) y sus colaboradores constataron así que la mayoría de los participantes que habían informado de unos mayores niveles de bienestar religioso y espiritual (en el sentido mencionado, de conexión con un poder superior) eran los que presentaban también mejores resultados en sus rehabilitaciones emocional y física. Se puede inferir que esta relación con un ser superior tiene beneficios a nivel neuropsicológico, pues permite incluso que pacientes con padecimientos graves a nivel cerebral presenten una mejor rehabilitación que aquellos que no tienen esta conexión o no practican la espiritualidad.

Lo anterior se vincula con propuestas como la de Davidson (2012) quien reconoce atributos como la compasión dentro de la función neuropsicológica empatía como evidencia de un cerebro saludable. Dichas conclusiones son producto de estudios de imagen cerebral, tareas ejecutivas en sujetos con prácticas religiosas en diferentes culturas.

\section{Referencias bibliográficas}

Arsuaga, J. y Martínez, I. (1998). La especie Elegida. Barcelona: Temas de Hoy ediciones.

Byrd, R. (1988) Positive Therapeutic Effects of Intercessory Prayer in a Coronary Care Unit Population. Southern medical journal, 81(7).

Calle, D. (2012) La etología como punto de partida epistémico frente a las formas de determinismo biológico. Ludus Vitalis, XX(37). 137-149

Calle, D. (2017) Filogenia y Desarrollo de Funciones Ejecutivas. Psicogente, 20(38), 368-381. http://doi. org/10.17081/psico.20.38.2557

Calle, D. y Conzáles, J. (2016). Funciones ejecutivas, empatía y conducta suicida: lo que nos cuenta la neurociencia de la cognición social. En: ]. Andrade et al, Ensayos Académicos en torno al suicidio Armenia: Fundación Participar Ediciones.

Calle, D.; Grañana, N.; Jiménez, M. y Romero, A. (2018) Aproximaciones a la caracterización neuropsicológica de la primera infancia en Colombia. Tunja: Editorial UPTC.

Carrasco, Y. (2015) La religión y su influencia en las conductas de salud (Tesis doctoral). Huelva: Universidad de Huelva.

Cencini, A. y Manenti, A. (2015). Psicología y Teología. Bologna: Editorial Salterrae.

Coppens, Y.; Grolier, E.; Pélicier, Y.; Reeves, H.; y Reisse, J. (1988) Les Origines. París: L Harmattan Ediciones. 
Davidson, R. (2012). The emotional life of your brain. Los Angeles: Penguin Publishing.

Durkheim, E. (1915). Las Formas Elementales de la Vida Religiosa. Akal Ediciones.

Estrada, S. (1992) La noción de principios y valores en la jurisprudencia de la corte constitucional. Revista Facultad de Derecho y Ciencias Políticas, 41(114), 41-76.

Flores, S.; Huerta, Y.; Herrera, O.; y Alonso, O. (2012) Factores Familiares y religiosos en la depresión en adultos mayores. Journal of Behavior, Health \& Social Issues, 3(2), 89-100.

Humphreys, J. (2004) Spirituality and Distress In Sheltered Battered Women. Journal of nursing scholarship, 32(3), 273-278 https://doi.org/10.1111/j.1547-5069.2000.00273.x

Lima, C. (2008). Psicología de la religión: una revisión científica. Ciências da Religião, História e Sociedade, 6(2).

Ments, L., Roelofsma, P. y Treur, J. (2018) Modeling the effect of religión on Human Empathy based on an adaptive temporal-causal network model Computational Social Networks, 5(1)

Moreira-Almeida, A., Koeing, H. y Luchetti, G. (2014) Clinical implications of spirituality to mental health: Review of evidence and practical guidelines. Revista Brasileira de Psiquiatria, 36(2), 176-82

Mundigo, A. (2005) Religión y salud reproductiva: encrucijadas y conflictos. Manchester: Center for Health and Social Policy.

Premack, D. \& Woodruff, G. (1978). Does the chimpanzee have a "theory of mind"? Behavior and brain sciences, 4, 515-526

Senter, Ky Caldwell, K. (2002) Spirituality and the Maintenance of Change: A Phenomenological Study of Women Who Leave Abusive Relationships. Contemporary Family Therapy, 24(4).

Rizzolatti, G. y Craighero, L. (2004). The Mirror-Neuron system. Annual Review of Neuroscience, 27.

Tomasello, M. (2016) Historia Natural de la Moralidad Humana. Bogotá: Editorial Universidad de los Andes.

Simkin, H. (2017) La Salud Mental en la Psicología de la religión y la espiritualidad. Revista científica arbitrada de la fundación Mente Clara, 2(2).

Thoresen, C. y Harris, A. (2002) Spirituality and Health: What's the Evidence and What's Needed? Annals of Behavioral Medicine, 24(1), 3-13.

Willson, E. (2012) La conquista social de la tierra. Barcelona: Debate.

Zegarra-Valdivia, J. y Chino, B. (2016) Impacto de la religión en la toma de decisiones y el funcionamiento ejecutivo. Revista de Psicología, 6(2); 57-73.

Nota.

' Este artículo hace parte de la primera fase de investigación "Aproximación al fenómeno religioso en Medellín: su impacto en la construcción de ciudad" 